\title{
Electrosprayed core-shell microspheres for protein delivery
}

\author{
Yiquan Wu, ${ }^{\text {acd }}$ I-Chien Liao, ${ }^{e}$ Scott J. Kennedy, ${ }^{c d}$ Jinzhi Du,${ }^{f}$ Jun Wang ${ }^{f}$ \\ Kam W. Leong ${ }^{e}$ and Robert L. Clark ${ }^{b c d}$
}

Received 22nd March 2010, Accepted 5th May 2010

First published as an Advance Article on the web 20th May 2010

DOI: $10.1039 / \mathbf{c 0 c c 0 0 5 3 5 e}$

This communication describes a single-step electrospraying technique that generates core-shell microspheres (CSMs) with encapsulated protein as the core and an amphiphilic biodegradable polymer as the shell. The protein release profiles of the electrosprayed CSMs showed steady release kinetics over 3 weeks without a significant initial burst.

The encapsulation of therapeutic agents in polymeric core-shell microspheres (CSMs) could serve as an efficient way to deliver various drugs because the shell could protect the therapeutic agents from degradation while allowing sustained release over time. ${ }^{1}$ Therefore, there has been tremendous interest in the fabrication of polymeric core-shell structured vectors with controllable microstructures and tailored properties for various biomedical applications. ${ }^{2}$ A number of approaches have been developed for preparing polymeric CSMs such as surface polymerization, layer-by-layer deposition, cross-linking of polymeric micelles, emulsion solvent evaporation, and vapor deposition polymerization. ${ }^{3}$ Of these, emulsion solvent evaporation has been a particularly popular technique for preparing micrometre scale polymeric CSMs. ${ }^{4}$ Recently, electrospraying, a technique in which a solution is pumped through a nozzle to create aerosol jets under an electrostatic field, has emerged as a cost effective and versatile technique to produce microstructures for the delivery of therapeutic agents. ${ }^{5}$ One outstanding feature of electrospraying is its ability to generate monodisperse droplets whose size may vary by as much as hundreds of micrometres to as little as tens of nanometres by optimizing the processing parameters.

This communication relates the first demonstration of electrospraying employed to generate polymeric CSMs loaded with protein using PCL-PPE-EA [poly( $\varepsilon$-caprolactone)-polyaminoethyl ethylene phosphate] amphiphilic polymer. The block length of PCL- $b$-PPE-EA was 100 and 20 for PCL and PPE-EA block, respectively, and the molecular weight of each

\footnotetext{
${ }^{a}$ Materials Science Program, Department of Mechanical Engineering, University of Rochester, Rochester, New York 14627, USA. E-mail:wuyiquan@me.rochester.edu

${ }^{b}$ Department of Biomedical Engineering, University of Rochester, Rochester, New York 14627, USA

${ }^{c}$ Department of Mechanical Engineering and Material Science,

Duke University, Durham, NC 27708, USA

${ }^{d}$ Center for Biologically Inspired Materials \& Material Systems, Duke University,

Durham, NC 27708, USA

${ }^{e}$ Department of Biomedical Engineering, Duke University, Durham, NC 27708, USA

${ }^{f}$ Hefei National Laboratory for Physical Sciences at Microscale and School of Life Sciences, University of Science and

Technology of China, Hefei, Anhui 230027, People's Republic of China
}

block was 11400 and 4080. The longer block length of PCL facilitates the dissolving of the block copolymer in dichloromethane (DCM) solution and contributes to the stability of the emulsion. Moreover, this block length of PPE-EA possesses sufficient positive charge to bind the proteins. PCL [poly( $\varepsilon$-caprolactone)] is a good candidate for drug delivery applications due to characteristics that include suitable mechanical properties, biodegradability and non-toxicity. ${ }^{6}$ Previous investigations on the preparation of PCL drug delivery carriers via electrospraying used a mixed solution where the drugs were directly dissolved into the organic solvents. ${ }^{7}$ However, there are several problems associated with the process, most notably: (1) hydrophilic substances (peptides, enzymes, proteins, and DNA) are difficult to encapsulate into hydrophobic PCL particles due to the immiscible combination of PCL oil solution and the protein aqueous solution, and (2) encapsulated biological substances can be easily degraded in harsh environmental conditions (e.g., the high temperatures used in spray-drying). The new technique overcomes the two limitations by forming CSMs at room temperature using an amphiphilic polymer with a hydrophilic block. In our process, an amphiphilic block copolymer (PCL-PPE-EA) was synthesized and then electrosprayed into particles with a coreshell structure. The advantage of the developed process is that the therapeutic delivery spheres prepared through electrospraying can be tuned to have wide ranges of particle sizes with narrow size distributions by optimizing the physical and chemical properties of precursor solution and the processing parameters. $^{8}$ Additionally, this process would provide an innovative and cost-effective way of preparing micro-scaled core-shell structured polymer particles with the ability of encapsulating hydrophilic agents in the core and hydrophobic agents in the shell. The technique could possibly be scaled up to serve pharmaceutical industry needs by developing appropriate tools to automate steps in the manufacturing process from the preparation of solution to the fabrication process.

BSA (bovine serum albumin, A2153, Sigma) (40\% w/v) or FITC-dextran $(40 \% \mathrm{w} / \mathrm{v})$ (Aldrich, FD4, average mol. weight 3000-5000) were prepared by dissolving the BSA protein or dextran in distilled water. The PCL-PPE-EA was dissolved in dichloromethane (DCM) at a concentration of $10 \% \mathrm{w} / \mathrm{v}$. Then, the aqueous BSA or dextran solution was dispersed drop by drop into the DCM solution containing PCL-PPE-EA copolymer to a concentration of $10 \% \mathrm{w} / \mathrm{w} \mathrm{BSA} /$ polymer under stirring to produce water-in-oil $(\mathrm{W} / \mathrm{O})$ emulsion. In the second step, the stable $\mathrm{W} / \mathrm{O}$ emulsion was electrosprayed to generate PCL-PPE-EA CSMs. The electrospraying voltage was $10 \mathrm{kV}$; the solution flow rate was fixed at $3.0 \mathrm{~mL} \mathrm{~h}^{-1}$, and the working distance was at $45 \mathrm{~cm}$. As a control, mixed 
solutions containing PCL and BSA in DCM solvent were also electrosprayed to generate spheres. An illustration of the formation of CSMs is presented in Fig. 1(a). The microstructures and morphologies of electrosprayed polymeric particles were characterized using a field-emission scanning electron microscope (FE-SEM, Philips XL 30 SEM). The distribution of particle diameters was determined using ImageJ analysis software (NIH). The PCL-PPE-EA particles encapsulated with FITC-dextran were characterized using an LSM 510 Zeiss confocal inverted microscope (CLSM). A transmission electron microscope (TEM, Hitachi HF-2000) was also employed to characterize the CSMs. The sizes of the micelles in the emulsion solution were characterized using dynamic light scattering (DLS, Wyatt Technology Corp, CA). To characterize the CSMs' ability to deliver protein, BSA loaded microspheres were deposited onto a $1 \mathrm{~cm}^{2}$ glass slide (approximate $0.25 \mathrm{mg}$ materials on the slide) and incubated in $1 \mathrm{~mL}$ of phosphate buffered saline (PBS) at $37{ }^{\circ} \mathrm{C}$. At predetermined time points, the PBS solution supernatants were collected and the protein concentration was measured using a microBCA protein assay (Pierce) at an absorbance wavelength of $562 \mathrm{~nm}$. The estimated BSA encapsulated amount was approximately 90\% for PCL-PPE-EA CSM. The percentage of material loss in the electrospraying process depends on the size of the collector. In our experiment, $85 \%$ of the material was delivered onto the collector.

For the PCL-PPE-EA solution, water-in-oil (W/O) emulsion formed when the aqueous solution of BSA was dispersed into DCM containing PCL-PPE-EA copolymer under stirring and the mixed PCL-PPE-EA solutions became turbid. However, for the PCL solution it remained clear when BSA was directly added and mixed with DCM solvent. Fig. 1(b) shows the size distributions of stabilized water droplets formed in the
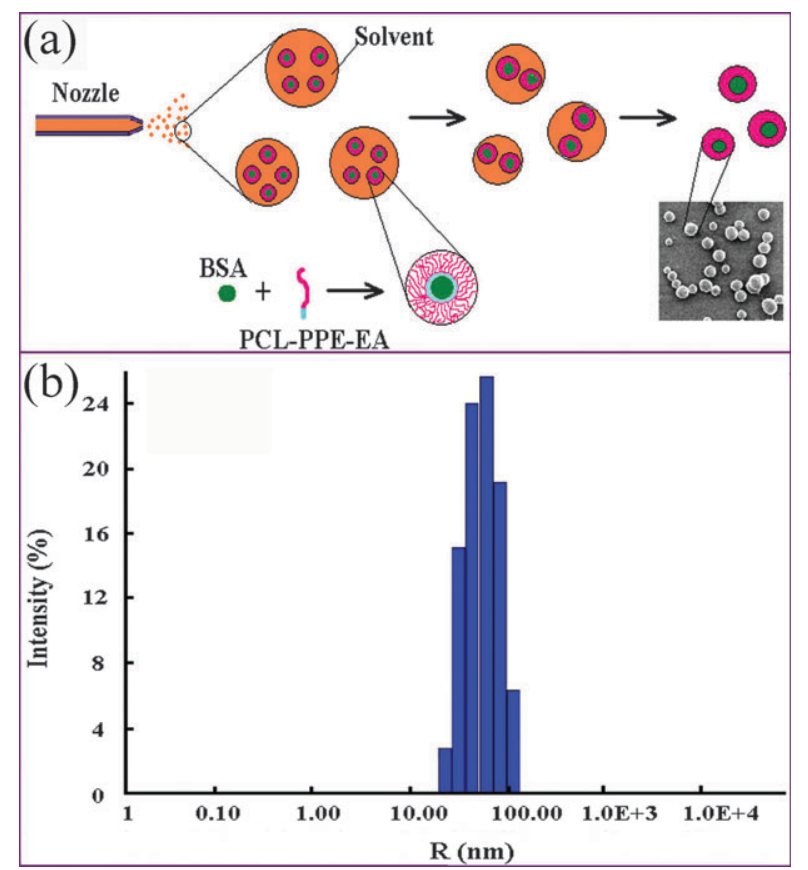

Fig. 1 (A) Schematic representation of the formation of coreshell structured particles through electrospraying an emulsion solution. (B) Size distributions of stabilized water droplets formed in oil emulsion.
$\mathrm{W} / \mathrm{O}$ emulsion. The DLS results show that stabilized water droplets had a narrow size distribution in the solution, with an average size of $58.6 \mathrm{~nm}$. Fig. 2(a) and (b) show an SEM image of PCL-PPE-EA CSMs created by electrospraying an emulsion solution directly onto the collector. The diameters of the majority of particles were distributed in a range of 2-8 $\mu \mathrm{m}$, with an average size of $3.0 \mu \mathrm{m}$. Fig. 2(c) and (d) show an SEM image of PCL particles prepared from a mixture solution of PCL polymer and BSA protein in dichloromethane solvent. The particles had diameters in a range of $1-10 \mu \mathrm{m}$, with an average size of $7.0 \mu \mathrm{m}$. The experiment demonstrated that under optimized processing parameters, the PCL and PCL-PPE-EA polymeric particles prepared by electrospraying have smooth surfaces and uniform spherical morphologies. The particle size distributions of PCL-PPE-EA CSMs show that most of the particle sizes were distributed in a range of 2-4 $\mu \mathrm{m}$, reaching a fraction $>70 \%$. The average particle diameter was $3.0 \mu \mathrm{m}$. However, for the electrosprayed PCL spheres, large particles were formed and the particle size distribution was wide. The particle size distribution scatters in a range of $0-12 \mu \mathrm{m}$, and the distribution of particles between 2 and $4 \mu \mathrm{m}$ only made up $33 \%$ of the sample.

The electrosprayed solid PCL spheres and PCL-PPE-EA CSMs were also characterized by SEM, TEM, and CLSM. Fig. 3(a) shows an SEM image of a fractured PCL particle electrosprayed from a mixed solution of PCL and BSA in DCM; it has a solid internal microstructure. In contrast, freeze-fractured PCL-PPE-EA CSM reveals clear core-shell microstructure, as shown in Fig. 3(b). The core-shell structures of the PCL-PPE-EA particles were further verified by TEM and CLSM images. The TEM image in Fig. 3(c) reveals two distinct components of the CSM: a lighter core of approximately $2 \mu \mathrm{m}$ and a darker shell of approximately $7 \mu \mathrm{m}$. The core within the CSMs was formed by agglomerating the small stabilized water droplets formed in oil emulsion during the electrospraying process. The CSMs were also visualized by confocal microscopy. The Nile red dye present in the polymer solution fluoresces within the shell section of

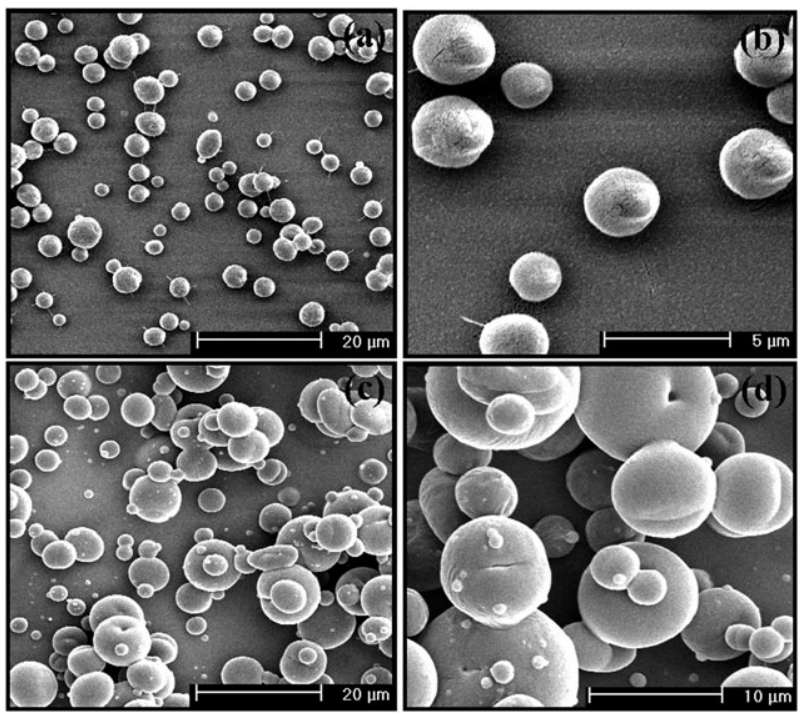

Fig. 2 SEM images of PCL-PPE-EA core-shell structured particles $(a, b)$ and PCL particles $(c, d)$ generated by electrospraying. 
the PCL-PPE-EA particles while the FITC-dextran present in the BSA aqueous solution illustrates the presence of a core, as shown in Fig. 3(d). All of these results further confirmed that electrospraying is capable of preparing core-shell structured polymer particles with the ability to encapsulate hydrophilic agents in the core part by using an amphiphilic copolymer. The observations by TEM and confocal microscopy indicated there is one large core compartment within each CSM. The BSA protein release profiles for the PCL microspheres and the PCL-PPE-EA CSMs demonstrated that the electrosprayed PCL-PPE-EA particles gradually released the BSA protein over a period of 20 days (Fig. 3e) without a significant initial burst. The PCL microspheres did not release BSA protein after the first day indicating no protein encapsulation within the materials, with a minute amount being adsorbed onto the surface. It was anticipated that the BSA protein release from
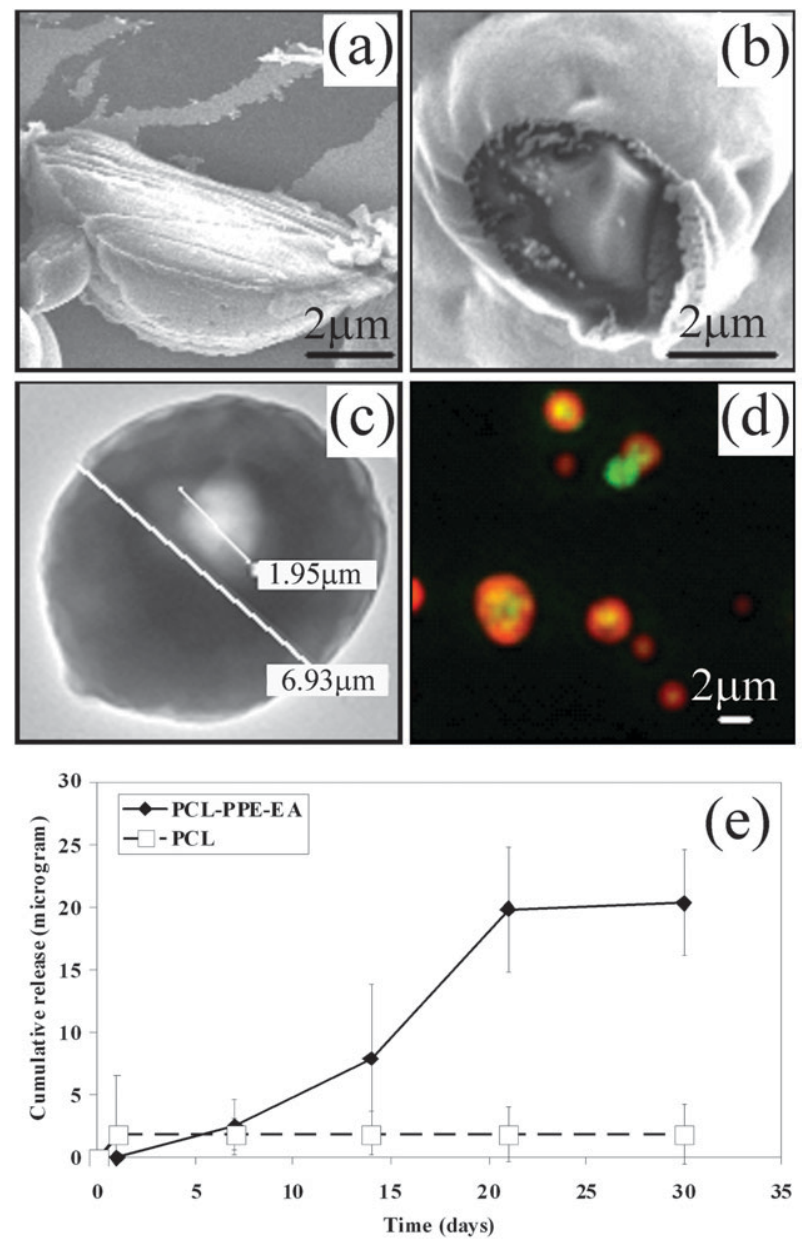

Fig. 3 An SEM image of a fractured solid PCL particle (a), an SEM image of a fractured PCL-PPE-EA core-shell structured particle (b), a TEM image of a PCL-PPE-EA core-shell particle (c), and a confocal image of PCL-PPE-EA core (green)-shell (red) particles (d). Scale bar: $2 \mu \mathrm{m}$. (e) BSA release profile from PCL particles and core-shell structured PCL-PPE-EA particles. these CSMs would be based on a diffusion process through the pores in the polymer matrix of CSMs. This result demonstrates the feasibility of using electrosprayed CSMs to deliver protein in a sustained manner.

In summary, polymeric CSMs with a gradual release profile were prepared using a simple electrospraying technique. The polymeric CSMs generated from electrospraying amphiphilic block copolymers are intriguing for therapeutic agent delivery vectors because they provide an effective means to encapsulate and release therapeutic agents. Moreover, this technique could provide a useful platform for the fabrication of other effective delivery vectors for healthcare applications.

This work was supported with NIH Grant (HL89764) and NSF Grants (CMMI 0609265 and DGE 0221632).

\section{Notes and references}

1 (a) X. Q. Gong, S. L. Peng and W. J. Wen, et al., Adv. Funct. Mater., 2009, 19, 292-297; (b) T. L. U. Nguyen, B. Farrugia and T. P. Davis, et al., J. Polym. Sci., Part A: Polym. Chem., 2007, 45, 3256-3272; (c) C. D. Jones and L. A. Lyon, Macromolecules, 2000, 33, 8301-8306; (d) V. R. Babu, M. Sairam and K. M. Hosamani, et al., Int. J. Pharm., 2006, 325, 55-62; (e) T. Basinska, Macromol. Biosci., 2005, 5, 1145-1168.

2 (a) S. Slomkowski, Prog. Polym. Sci., 1998, 23, 815-874; (b) K. Sparnacci, M. Laus and L. Tondelli, et al., J. Biomater. Sci., Polym. Ed., 2005, 16, 1557-1574; (c) L. Chiarantini, A. Cerasi and E. Millo, et al., J. Controlled Release, 2005, 101, 397-398; (d) X. Q. Gong, S. L. Peng and W. J. Wen, et al., Adv. Funct. Mater., 2009, 19, 292-297; (e) Z. H. Gan, T. F. Jim and M. Li, et al., Macromolecules, 1999, 32, 590-594.

3 (a) Z. Li, J. F. Ding and M. Day, et al., Macromolecules, 2006, 39, 2629-2636; (b) W. H. Li and H. D. H. Stover, Macromolecules, 2000, 33, 4354-4360; (c) C. Lemarchand, P. Couvreur and M. Besnard, et al., Pharm. Res., 2003, 20, 1284-1292; (d) H. S. Yoon, M. J. Choi and K. A. Lee, et al., Macromol. Res., 2008, 16, 85-102; (e) K. Ishizu, Prog. Polym. Sci., 1998, 23, 1383-1408; $(f)$ Y. W. Zhang, M. Jiang and J. X. Zhao, et al., Macromolecules, 2004, 37, 1537-1543.

4 (a) Y. Miyazaki, S. Yakou and K. Takayama, Biol. Pharm. Bull., 2007, 30, 543-546; (b) S. R. Mao, Y. Shi and L. Li, et al., Eur. J. Pharm. Biopharm., 2008, 68, 214-223; (c) R. Atkin, P. Davies and J. Hardy, et al., Macromolecules, 2004, 37, 7979-7985.

5 (a) Y. Q. Wu, J. A. MacKay and J. R. McDaniel, et al., Biomacromolecules, 2009, 10, 19-24; (b) Y. Q. Wu and R. L. Clark, J. Colloid Interface Sci., 2007, 310, 529-535; (c) Y. Q. Wu and R. L. Clark, J. Biomater. Sci., Polym. Ed., 2008, 19, 573-601; (d) N. Arya, S. Chakraborty and N. Dube, et al., J. Biomed. Mater. Res., Part B, 2009, 88, 17031; (e) L. Y. Yeo, Z. Gagnon and H. C. Chang, Biomaterials, 2005, 26, 6122-6128; $(f)$ K. Pancholi, N. Ahras and E. Stride, et al., J. Mater. Sci.: Mater. Med., 2009, 20, 917-923.

6 (a) V. R. Sinha, K. Bansal and R. Kaushik, et al., Int. J. Pharm., 2004, 278, 1-23; (b) J. S. Chawla and M. M. Amiji, Int. J. Pharm., 2002, 249, 127-138; (c) S. R. Jameela, N. Suma and A. Jayakrishnan, J. Biomater. Sci., Polym. Ed., 1997, 8, 457-466.

7 (a) L. Ding, T. Lee and C. H. Wang, J. Controlled Release, 2005 , 102, 395-413; (b) T. Ciach, J. Drug Delivery Sci. Technol., 2007, 17, 367-375; (c) J. W. Xie, J. C. M. Marijnissen and C. H. Wang, Biomaterials, 2006, 27, 3321-3332.

8 (a) A. M. Ganan-Calvo, J. Davila and A. Barrero, J. Aerosol Sci., 1997, 28, 249-275; (b) Y. Q. Wu and R. L. Clark, Electrohydrodynamic Processing of Micro and Nanometer Biomaterials. Handbook of Fabrication and Processing of Biomaterials, CRC Press/Taylor and Francis Group, Boca Raton, FL, 2008, pp. 275-333. 